\title{
Diagnostic Value of the Visual Evoked Potential Investigation in Optic Neuritis
}

\author{
Gavkhar Kh. Khamraeva*; Khalidjan M. Kamilov, PhD, ScD; \\ Munurakhon S. Kasimova, $\mathrm{PhD}, \mathrm{ScD}$ \\ Tashkent Institute of Postgraduate Medical Education \\ Tashkent, Uzbekistan
}

\begin{abstract}
Objective: Comparative analysis of the results of the visual evoked potential investigation according to stages of optic neuritis $(\mathrm{ON})$.

Material and Methods: The study included 90 patients (90 eyes) with different stages of ON. A control group comprised 10 somatically healthy persons (10 eyes) of the same age without ophthalmic pathology. All examined underwent standard ophthalmologic examination, including visometry, tonometry, perimetry, and ophthalmoscopy, as well as optical coherence tomography (OCT) and visual evoked potentials (VEP) determination.

Results: The VEP parameters provide identification of the functional disorders of the optic nerve. Our results showed that there is delayed latency and reduced amplitude of the P100 component in patients with ischemic and atrophic stages of the disease. In ON, P100 was the most informative index. We noted that the N145 parameter in ON is not very informative.

Conclusion: An estimation of VEP parameters provides a more reliable diagnosis of the stage of disease and aids in monitoring the effectiveness of treatment. (Int J Biomed. 2015;5(3):147-150.)
\end{abstract}

Keywords: optic neuritis; visual evoked potentials (VEPS); optical coherence tomography (OCT).

\section{Introduction}

Many clinicians emphasize the importance of the study of visual evoked potentials (VEPs) to evaluate visual pathway function and obtain information on the functional status of the axons and myelin sheath of the optic nerve [1-3]. VEPs represent the visual pathway from the retina along the optic nerve, optic chiasma, optic tract, and optic radiation to the occipital optical cortex [4-7].

VEPs registration is a valuable diagnostic test in the evaluation of patients with ON. Verification of the visual pathway lesion is particularly important in the absence of changes in the fundus in patients with retrobulbar neuritis and early stages of inflammation of the optic nerve [8-14]. Despite the large number of articles devoted to this issue, the dynamics of changes in VEPs in ON are still under discussion.

*Corresponding author: Gavkhar Kh. Khamraeva. Tashkent Institute of Postgraduate Medical Education, Tashkent, Uzbekistan. E-mail: gavhar08021982@mail.ru
Objective: Comparative analysis of the results of the visual evoked potential investigation according to stages of optic neuritis $(\mathrm{ON})$.

\section{Material and Methods}

The study included 90 patients (90 eyes) with different stages of ON of an inflammatory etiology. A control group comprised 10 somatically healthy persons $(10$ eyes $)$ of the same age without ophthalmic pathology. Exclusion criteria included conjunctivitis, uveitis, glaucoma, ON of a demyelinating etiology, as well as the degenerative, ischemic, vascular and oncological diseases of the eye.

All examined underwent standard ophthalmologic examination, including visometry, tonometry, perimetry, and ophthalmoscopy, as well as optical coherence tomography (OCT) and VEP determination. VEPs were performed with a Neuron-Spectrum-4 EPM apparatus by presenting an alternating chessboard pattern and single light pulses.

Patients were distributed in groups according to the classification of $\mathrm{ON}$, depending on the condition of the optic 
disk (OD) [13]. Group 1 included 28 patients (28 eyes) with hyperemia of the OD, Group 2 included 21 patients ( 21 eyes) with edema of the OD, Group 3 included 26 patients (26 eyes) with ischemia of the OD, and Group 4 included 15 patients (15 eyes) with glial atrophy of the OD.

The study was conducted in accordance with ethical principles of the Declaration of Helsinki. It was approved by the Tashkent Institute of Postgraduate Medical Education Ethics Committee. Written informed consent was obtained from all participants.

Statistical analysis was performed using the statistical software Statistica for Windows 6.0. Differences of continuous variables with a normal distribution (presented as $\mathrm{M} \pm \mathrm{SEM}$ ) between the two groups were calculated using the independent-sample t-test A probability value of $P<0.05$ was considered statistically significant.

\section{Results and Discussion}

The visual acuity values were $0.62 \pm 0.05,0.31 \pm 0.06$, $0.22 \pm 0.04$, and $0.03 \pm 0.01$ in Groups 1, 2, 3, and 4, respectively. The anterior segment of the eyeball was unchanged in biomicroscopy.

During direct ophthalmoscopy, in Group 1 we noted hyperemia of the OD, lack of clarity in borders along the vessels, and an increase to $18.4 \pm 2.0$ in the number of vessels passing through the edge of the disc; arteries had normal caliber, veins were extended, and macular reflex was saved. During OCT we observed normal levels of thickness of the retinal nerve fiber layer (RNFL) and the OD.

In Group 2, the OD was sharply hyperemic, borders were unclear, and the number of vessels passing through the edge of the disc was increased to $22.4 \pm 2.0$; there was marked swelling of the OD and peripapillary zone, arteries and veins were dilated, and macular reflex was reduced. We observed an increased RNFL thickness in OCT, as well as the area of the OD.

In Group 3, the OD was pale and edematous with fuzzy borders, and the number of vessels passing through the edge of the disc was $14.2 \pm 2.0$; we marked swelling of the peripapillary zone, narrowed arteries and extended veins, and a reduction in macular reflex. In OCT, RNFL thickness was significantly reduced in comparison with Group $2(P<0.01)$ (Table 1).

In Group 4, the OD was pale, borders along the vessels were unclear, the number of vessels passing through the edge of the disc was $11.3 \pm 2.0$, the arteries and veins were narrowed, and macular reflex was absent. The average RNFL thickness was significantly lower compared to the values in Groups 2 and $3(P<0.001)$. Parameters of the rim area and the area of the OD had no significant differences in comparison with other groups (Table 1).

When VEPs checkerboard stimulus was used, peak latencies of N75 (1 st channel) significantly increased in Group $1(78.26 \pm 1.04 \mathrm{~ms})$ compared with the control group $(75.2 \pm 0.2$ $\mathrm{ms} ; P<0.05)$. The prolonged latency evidenced reduced impulse conduction along the visual pathways. There was also a statistically significant increase in the latency of N75 in Group $2(81.64 \pm 2.09 \mathrm{~ms})$ compared to control $(P<0.05)$.
In Group 3, this parameter increased up to $93.14 \pm 3.57 \mathrm{~ms}$ and was statistically greater vs. Group $2(P<0.01)$, but a significant difference compared with Group 4 ( $98.22 \pm 6.38 \mathrm{~ms})$ was not detected. The prolonged latency indicates the defeat of the myelin sheath, which is associated with the reduced impulse conduction along the visual pathways.

Table 1.

Comparative characteristics of the morphometric parameters of the $O D$

\begin{tabular}{|c|c|c|c|c|}
\hline \multicolumn{1}{|c|}{ Group } & Rim Area & Disc Area & RNFL & Cup Volume \\
\hline \multirow{3}{*}{ Group 1 } & $1.65 \pm 0.08$ & $2.00 \pm 0.07$ & $105.7 \pm 2.51$ & $0.07 \pm 0.02$ \\
\cline { 2 - 5 } & $P 1>0.05$ & $P 1>0.05$ & $P 1>0.05$ & $P 1<0.05$ \\
& $P 2<0.001$ & $P 2<0.01$ & $P 2<0.001$ & $P 2<0.01$ \\
\hline \multirow{3}{*}{ Group 2 } & $2.70 \pm 0.25$ & $2.60 \pm 0.16$ & $295.38 \pm 19.15$ & $0.003 \pm 0.002$ \\
\cline { 2 - 5 } & $P 1<0.01$ & $P 1>0.05$ & $P 1<0.001$ & $P 1<0.001$ \\
& $P 3>0.05$ & $P 3<0.05$ & $P 3<0.001$ & $P 3>0.05$ \\
\hline \multirow{3}{*}{ Group 3 } & $2.28 \pm 0.19$ & $2.12 \pm 0.10$ & $142.83 \pm 8.15$ & $0.004 \pm 0.003$ \\
\cline { 2 - 5 } & $P 1<0.05$ & $P 1>0.05$ & $P 1<0.01$ & $P 1<0.001$ \\
& $P 4<0.01$ & $P 4<0.05$ & $P 4<0.001$ & $P 4<0.01$ \\
\hline \multirow{3}{*}{ Group 4 } & $1.53 \pm 0.08$ & $1.74 \pm 0.10$ & $92.99 \pm 2.73$ & $0.03 \pm 0.008$ \\
\cline { 2 - 5 } & $P 1>0.05$ & $P 1>0.05$ & $P 1>0.05$ & $P 1<0.05$ \\
\hline Control group & $1.48 \pm 0.29$ & $2.26 \pm 0.5$ & $104.5 \pm 6.9$ & $0.24 \pm 0.02$ \\
\hline
\end{tabular}

P1 - vs. the control group; P2 - Group 1 vs. Group 2; P3 - Group 2 vs. Group 3; P4 - Group 3 vs. Group 4.

In assessing the form of the P100 component, the impairment of the VEP configuration in the form of P100 splitting was detected. The W-like shape of P100 peak reflected the presence of a central scotoma in the visual field or partial atrophy of the optic nerve, which was confirmed by other researchers $[5,10]$.

Almost all the subjects showed an increase in P100 latency from 10 to $30 \mathrm{~ms}$ compared to the upper limit of normal values upon checkerboard pattern stimulus. In Group 1 , this parameter was $109.86 \pm 2.11 \mathrm{~ms}$, which was significantly higher vs. the control group $(P<0.05)$. In the stages of edema and ischemia, this parameter tended to increase, and amounted to an average of $112.73 \pm 3.52 \mathrm{~ms}(P<0.05)$ and $123.65 \pm 3.93$ ms $(P<0.05)$, respectively. We also registered an increase in latency in Group 4 by $20 \%$ to $25 \%$; the average value of latency was $127.24 \pm 7.34 \mathrm{~ms}(P<0.05)$ and ranged from 118 to $135 \mathrm{~ms}$.

In the analysis of the N145 component in 1 and 2 channels, we did not find any significant differences between the patient groups and control. The parameters of the latency of the VEPs are shown in Table 2. Evaluation of latency of the N75 and P100 components (in 2nd channel) in patients with different stages of ON revealed a statistically significant increase in the estimated parameters $(P<0.05)$.

Analysis of N75-P100 amplitude revealed a significant increase in this indicator in Groups 1 and 2 compared with the control group: $13.91 \pm 0.79 \mu \mathrm{V}$ and $15.77 \pm 1.29 \mu \mathrm{V}$, respectively, and $P<0.05$ in both cases. Ischemia, toxic effects of exudates, compression of nerve fibers, and disintegration of the myelin sheath of nerve fibers occur in the acute phase of neuritis. Increasing the amplitude of the N75-P100 component apparently was connected with the reactive stimulation of 
axons of the optic nerve due to exudative reactions occurring in the shell of the optic nerve. The amplitude of the N75-100 component was significantly decreased in Group $3(6.76 \pm 0.76$ $\mu \mathrm{V})$ compared with the control group $(P<0.05)$, as well as with Group $2(P<0.001)$. The reduction of VEP amplitude is associated with the blockage of pulse conduction, probably due to the damage of the axial cylinders of axons. Reduction of amplitude indicates a decrease in the number of functioning axons. A significant reduction in the amplitude of the N75-P100 component $(3.68 \pm 0.4 \mu \mathrm{V}, P<0.05)$ was observed in Group 4 (patients with OD atrophy). The reduction in VEP amplitude is correlated with changes in visual functions $(\mathrm{r}=0.42)$, which indicates the death of nerve fibers. Changes in VEP amplitude are presented in Table 3. Analysis of the amplitude of the P100-N145 component revealed a significant increase in this indicator in Groups 1 and 2 compared with the control group: $12.73 \pm 1.06 \mu \mathrm{V}$ and $13.31 \pm 0.94 \mu \mathrm{V}$, respectively, and $P<0.05$ in both cases. The amplitude of the P100-N145 component was significantly decreased in Group $3(7.54 \pm 0.83 \mu \mathrm{V})$ compared to Group $2(P<0.001)$. No significant differences, compared to the control group, were detected. A significant reduction in the amplitude of the P100-N145 component was observed in Group 4 (patients with OD atrophy; $3.67 \pm 0.66 \mu \mathrm{V}$ ) vs. the control group $(P<0.05)$ and Group $3(\mathrm{P}<0.01)$.

Table 2.

The parameters of the latency of the VEPs

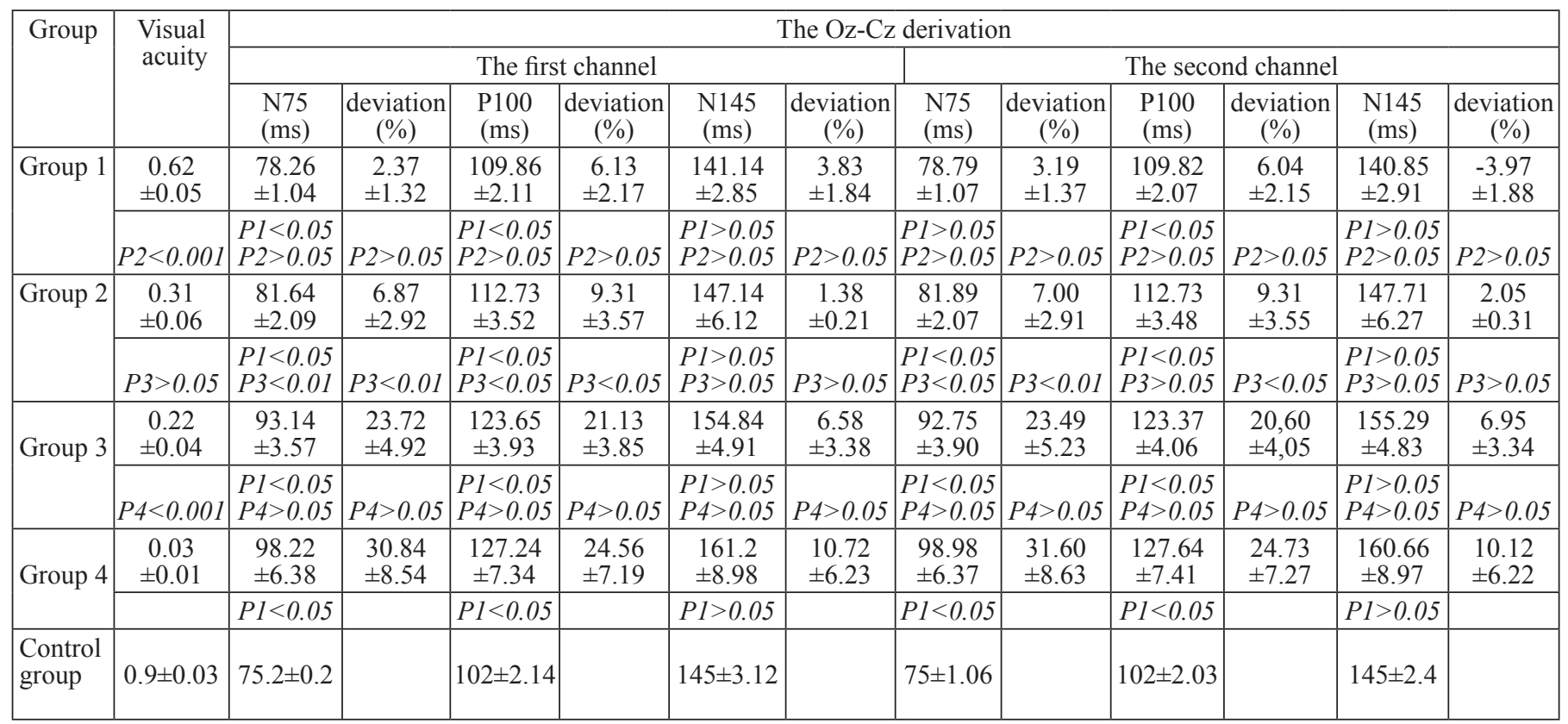

P1 - vs. the control group; P2 - Group 1 vs. Group 2; P3 - Group 2 vs. Group 3; P4 - Group 3 vs. Group 4.

Table 3.

The parameters of the amplitude of the VEPS

\begin{tabular}{|c|c|c|c|c|c|c|c|c|c|}
\hline \multirow[t]{3}{*}{ Group } & \multirow{3}{*}{$\begin{array}{l}\text { Visual } \\
\text { acuity }\end{array}$} & \multicolumn{8}{|c|}{ The $\mathrm{Oz}-\mathrm{Cz}$ derivation } \\
\hline & & \multicolumn{4}{|c|}{ The first channel } & \multicolumn{4}{|c|}{ The second channel } \\
\hline & & $\begin{array}{c}\text { N75-P100 } \\
(\mu \mathrm{V})\end{array}$ & $\begin{array}{c}\text { deviation } \\
(\%)\end{array}$ & $\begin{array}{c}\text { P100-N145 } \\
(\mu \mathrm{V})\end{array}$ & $\begin{array}{c}\text { deviation } \\
(\%)\end{array}$ & $\begin{array}{c}\text { N75-P100 } \\
(\mu \mathrm{V})\end{array}$ & deviation (\%) & $\begin{array}{c}\text { P100-N145 } \\
(\mu \mathrm{V})\end{array}$ & $\begin{array}{c}\text { deviation } \\
(\%)\end{array}$ \\
\hline Group 1 & $P 2<0.001$ & $\begin{array}{l}P 1<0.05 \\
P 2>0.05\end{array}$ & $P 2<0.01$ & $\begin{array}{l}P 1<0.05 \\
P 2>0.05\end{array}$ & $P 2>0.05$ & $\begin{array}{l}P 1<0.05 \\
P 2>0.05\end{array}$ & $P 2>0.05$ & $\begin{array}{l}P 1<0.05 \\
P 2>0.05\end{array}$ & $P 2>0.05$ \\
\hline \multirow[t]{2}{*}{ Group 3} & $0.23 \pm 0.04$ & $6.76 \pm 0.76$ & $-29.00 \pm 7.34$ & $7.54 \pm 0.83$ & $-6.55 \pm 1.41$ & $6.62 \pm 0.77$ & $-30.43 \pm 7.60$ & $7.07 \pm 0.71$ & $-9.72 \pm 7.81$ \\
\hline & $P 4<0.001$ & $\begin{array}{l}P 1<0.05 \\
P 4<0.01\end{array}$ & $P 4<0.01$ & $\begin{array}{l}P 1>0.05 \\
P 4<0.01\end{array}$ & $P 4<0.001$ & $\begin{array}{l}P 1<0.05 \\
P 4<0.01\end{array}$ & $P 4<0.05$ & $\begin{array}{c}P 1>0.05 \\
P 4<0.001\end{array}$ & $P 4<0.01$ \\
\hline Group 4 & $0.03 \pm 0.01$ & $3.68 \pm 0.46$ & $-57.92 \pm 6.78$ & $3.67 \pm 0.66$ & $-49.00 \pm 9.85$ & $3.60 \pm 0.49$ & $-56.39 \pm 8.16$ & $3.58 \pm 0.55$ & $-52.47 \pm 8.81$ \\
\hline
\end{tabular}

P1 - vs. the control group; P2 - Group 1 vs. Group 2; P3 - Group 2 vs. Group 3; P4 - Group 3 vs. Group 4. 


\section{Conclusion}

The VEP parameters provide identification of the functional disorders of the optic nerve. Our results showed that there is delayed latency and reduced amplitude of the P100 and N75 components in patients with ischemic and atrophic stages of the disease. In ON, P100 was the most informative index. We noted that the N145 parameter in ON is not very informative. An estimation of VEP parameters provides a more reliable diagnosis of the stage of disease and aids in monitoring the effectiveness of treatment.

\section{Competing interests}

The authors declare that they have no competing interests.

\section{References}

1. Krivosheev AA. Topographic mapping of visual evoked potentials in the diagnosis of diseases of the visual pathway. Abstract of PhD thesis. Moscow; 2008. [in Russian].

2. Shamshinova AM, Volkov VV. The functional methods in ophthalmology. Moscow: Meditsina, 2004. [in Russian].

3. Fraser CL, Klistorner A, Graham SL, Garrick R, Billson FA, Grigg JR. Multifocal visual evoked potential analysis of inflammatory or demyelinating optic neuritis. Ophthalmology. 2006;113(2):321-323.

4. Grover LK, Hood DC, Ghadiali Q, Grippo TM, Wenick AS, Greenstein VC, et al. A comparison of multifocal and conventional visual evoked potential techniques in patients with optic neuritis/multiple sclerosis. Doc Ophthalmol. 2008;
117(2):121-128.

5. Roceanu A, Romaniţan O, Antochi F, Tiu C, Băjenaru O, Pascu R, et al. Assessment of a patient with optic neuropathy. Oftalmologia. 2010;54:3-8. [Article in Romanian]

6. Osborne BJ, Volpe NJ. Optic neuritis and risk of MS: differential diagnosis and management. Cleve Clin J Med. 2009;76(3):181-190.

7. Almarcegui C1, Dolz I, Pueyo V, Garcia E, Fernandez FJ, Martin J, et al. Correlation between functional and structural assessments of the optic nerve and retina in multiple sclerosis patients. Neurophysiol Clin. 2010;40(3):129-35.

8. Kamilov KhM. Differentiated approach to the diagnosis of the edematous states of the optic disk. Tashkent: Fan va Technology, 2008. [in Russian].

9. Klistorner A, Fraser C, Garrick R, Graham S, Arvind H. Correlation between full-field and multifocal VEPs in optic neuritis. Doc Ophthalmol. 2008;116 (1):19-27.

10. Klistorner A, Arvind H, Nguyen T, Garrick R, Paine M, Graham S, et al. Multifocal VEP and OCT in optic neuritis: a topographical study of the structure-function relationship. Doc Ophthalmol. 2009;118(2):129-137.

11. Kolbe S, Chapman C, Nguyen T, Bajraszewski C, Johnston L, Kean M, et al. Optic nerve diffusion changes and atrophy jointly predict visual dysfunction after optic neuritis. Neuroimage. 2009;45:679-686.

12. Jindahra P1, Hedges TR, Mendoza-Santiesteban CE, Plant GT. Optical coherence tomography of the retina: applications in neurology. Curr Opin Neurol. 2010;23(1):16-23.

13. Zhaboedov GD, Skripnik RL. The lesions of the optic nerve. Kiev: Health, 2006. [in Russian].

14. Ionkina IV Clinical and electrophysiological peculiarities of optic neuritis and prediction of disease outcome. Abstract of PhD thesis. Yekaterinburg; 2013. [in Russian]. 